The level of antibodies to pneumococcal capsular polysaccharide was determined using the EIA PCP IgG kit (TestLine Clinical Diagnostics s.r.o., Czech Republic) before vaccination, 1, 3 and 12 months after vaccination.

Results: The dynamics of the concentration of antibodies to pneumococcal capsular polysaccharide in patients with SpA is presented in the Table 1.

Table 1. Concentration of pneumococcal antibodies, U/ml, Me [25; 75 percentile]

1 visit (initial) $\quad 2$ visit (after 1 month) 3 visit (after 3 month) 4 visit (after 12 month) $80.0[35.2 ; 154.0] \quad 160.1[73.5 ; 245.7]$ * $214.5[103.2 ; 255.0]$ ** $175.0[120.1 ; 260.1]$ *

${ }^{*} \mathrm{p}=0.01{ }^{* *} \mathrm{p}=0.005$

At 1, 3 and 12 months after vaccination, the concentration of antibodies to pneumococcal capsule polysaccharide was significantly higher compared to the baseline values. In $81 \%$ of patients, vaccination tolerance was good. Reactions at the injection site (pain, swelling and hyperemia of the skin up to $2 \mathrm{~cm}$ in diameter), resolved independently after 1-5 days, were observed in 6 patients. In 2 patients, a severe local reaction was registered in the form of pain in the arm, infiltration and hyperemia of the skin up to 8 and $15 \mathrm{~cm}$ in diameter, respectively, accompanied by low-grade fever in one patient for 2 days, and febrile fever in the other for 3 days. In both cases, these symptoms were completely stopped after administration of paracetamol and antihistamines. Exacerbation of SpA and the emergence of new autoimmune disorders were not detected. During the follow-up period, no patients developed lower respiratory tract infections. Patients suffering from frequent sinusitis and otitis reported the absence of these infections after vaccination.

Conclusion: The obtained data indicate satisfactory immunogenicity and good tolerability of PPV-23 in patients with SpA. Further studies are needed to better assess the immunogenicity and safety of vaccine, as well as to study of the influence of anti-rheumatic therapy on the effectiveness of immunization.

Disclosure of Interests: None declared.

DOI: 10.1136/annrheumdis-2021-eular.505

\section{POS1152 COMORBID INFECTIONS IN PATIENTS WITH SPONDYLOARTHRITIS.}

M. Baranova ${ }^{1}$, N. Muravyeva ${ }^{1}$, B. Belov ${ }^{1} .{ }^{1}$ V.A. Nasonova Research Institute of Rheumatology, Department of Study Comorbid Infection and Monitoring Antirheumatic Therapy, Moscow, Russian Federation

Background: Data on the frequency and structure of comorbid infections $(\mathrm{Cl})$ in spondyloarthritis ( $\mathrm{SpA})$ are few and contradictory.

Objectives: The aim of the study was to study the frequency and structure of $\mathrm{Cl}$ in the inpatient population of SpA patients in the course of a one-moment retrospective study.

Methods: The study included 208 patients with SpA (121 men, 87 women, mean age $39.1 \pm 12.2$ years) who were hospitalized at the V.A. Nasonova Research Institute of Rheumatology. Ankylosing spondylitis was diagnosed in 133 patients, psoriatic arthritis - in 57, spondyloarthritis associated with Crohn's disease - in 1 , undifferentiated spondyloarthritis - in 17. The majority of respondents had higher education $(60.6 \%)$. None of the patients consumed alcohol on a daily basis, 124 patients never smoked. The Charlson comorbidity index, equal to 0 , had 98 respondents, $1-51,2-27,3-15,4-10,5$ or more - 7. Most patients $(\mathrm{n}=168)$ received nonsteroidal anti-inflammatory drugs (NSAIDs), as well as glucocorticoids-GC (average duration of administration $239.5 \pm 65.8$ months), methotrexate-MT $(32.4 \pm 46.2)$, sulfasalazine $(21.0 \pm 32.1)$, leflunomide $(24.0 \pm 46.6)$, biological drugs - TNF-a inhibitors (21.5 \pm 23.3 ), inhibitors of interleukin (ilL)-12/23 (9.0 \pm 5.2$)$, ilL-17 (11.0 \pm 9.3$)$. Patients were interviewed by a research doctor with the completion of a unified questionnaire, additional data were obtained from medical documentation.

Results: Leading in the structure of $\mathrm{Cl}$ in patients with $\mathrm{SpA}$ were respiratory tract infections: acute nasopharyngitis $(n=168)$, tonsillitis $(74)$, acute bronchitis (34), sinusitis (33), pneumonia (29, including 9 cases by the virus SARSCoV-2), influenza (31), tuberculosis-TB (5, including 2 cases on infliximab therapy, which was the reason for withdrawal of the drug). Infectious diseases of other localization include herpes-viral infections $(n=109)$, mycoses (51), urinary tract infections (47), conjunctivitis and blepharitis (37), otitis (25), genital infections (15), skin infections (14), intestinal infections (13), COVID19 without lung damage (19), nervous system infections (2), bone infections (2), chronic hepatitis B (1), chronic hepatitis C (1), HIV infection (1), rubella in adulthood (1), measles in adulthood (1). $20.2 \%$ of patients reported more frequent development of $\mathrm{Cl}$ after the onset of the of SpA: 35 of them received immunosuppressive therapy, 7 - monotherapy of NSAIDs. In SpA patients receiving immunosuppressive drugs, there was an increase in the frequency of acute nasopharyngitis (more often 3 times a year), sinusitis, acute bronchitis, pneumonia and herpes-viral infections, in particular herpes zoster. $29.8 \%$ of patients reported a more severe course of $\mathrm{Cl}$ against the background of SpA (12 of them did not receive immunosuppressive drugs). Temporary discontinuation of therapy due to the development of $\mathrm{Cl}$ occurred in $26.4 \%$ of patients. At the same time, in 5 patients treated with GC (including in combination with MT, $n=3$ ), the development of furunculosis was the reason for changing the treat ment regimen. In one patient, MT therapy was discontinued due to the frequent development of purulent tonsillitis. Exacerbation of $\mathrm{SpA}$ after $\mathrm{Cl}$ was diagnosed in 84 patients (70 of them received immunosuppressive therapy).

Conclusion: The data obtained indicate the important of the problem of $\mathrm{Cl}$ in $\mathrm{SpA}$. Further studies are needed on large samples of patients in order to find significant risk factors for $\mathrm{Cl}$, study their relationship with clinical characteristics and influence on the course of SpA

Disclosure of Interests: None declared.

DOI: 10.1136/annrheumdis-2021-eular.551

\section{POS1153 1 EFFECTIVENESS OF SCREENING IN PATIENTS WITH RHEUMATIC DISEASE ON BIOLOGICAL THERAPY AND RISK OF ACTIVE TUBERCULOSIS}

S. Abdulaziz ${ }^{1}$, S. Attar ${ }^{2}$, W. Bajhammoh ${ }^{3}$, E. A. Sindi ${ }^{1}$, D. M. Ayish ${ }^{4}$, E. Bakhashwain ${ }^{5}{ }^{1}$ King Fahad Hospital, Medicine, Division of Rheumatology, Jeddah, Saudi Arabia; ${ }^{2}$ King Abdulaziz University, Medicine,Division of Rheumatology, Jeddah, Saudi Arabia; ${ }^{3}$ King Fahad Hospital, Medicine, Division of Infectious Diseases, Jeddah, Saudi Arabia; ${ }^{4}$ Prince Mohammed Bin Nasser Hospital, Medicine, Division of Rheumatology, Gizan, Saudi Arabia; ${ }^{5}$ East Jeddah Hospital, Medicine, Division of Rheumatology, Jeddah, Saudi Arabia

Background: Treatment with biologic therapy has been associated with a high risk of reactivation of latent tuberculosis (TB). Preventive strategies for tuberculosis remain a crucial step before initiating biologics in rheumatic disease.Treatment with biological therapy has been associated with high risk of reactivation of latent tuberculosis (TB). Prevention strategies remain a crucial step before initiating biologics.

Objectives: We aimed to assess the effectiveness of TB screening before the initiation of biologics and the risk of occurrence of active TB among patients with rheumatic diseases on biologic therapies.

The study aimed to access the effectiveness of TB screening recommendations before the initiation of biological therapy and identify the incidence of active TB among these patients.

Methods: We performed a hospital-based retrospective cohort study among rheumatic disease patients on biological therapy in two centers in Jeddah between January 2005 to December 2019. Medical files were retrospectively reviewed for demographics data, baseline screening for TB, use of prophylaxis, information on DMARDs and biological therapies, and outcomes results were collected.

Results: A total of 365 patients were included over a period of 14 years. Two hundred ninety-two (80\%) had Rheumatoid arthritis (RA), $13 \%$ psoriatic arthritis (PSA), $9 \%$ spondyloarthritis (SPA), $2 \%$ SLE, and $4 \%$ others. The mean age was $47.54( \pm 14.2), 311(85 \%)$ were females with a mean duration of disease 8.45 years $( \pm 6.58)$. Hundred forty-nine $(42.3 \%)$ were on steroids. Anti TNFs were prescribed in $213(58.4 \%)$ patients, Non Anti-TNFs 124 (36.6\%) patients, and Jak inhibitors $18(5 \%)$ patients.TB screening was done to all patients except 3 patients (data missing) before commencing biologics. Forty-four (12.1\%) patients had latent TB at baseline and all received chemoprophylaxis with isoniazid before starting biologics. Four patients with active TB were identified (one with Behcet's disease and three with RA). One patient had a reactivation of latent TB and 3 patients developed de novo TB. Three out of four had an infection in the first 6 months of treatment (one on infliximab and two on rituximab) and one case after 1 year of stopping adalimumab. Two cases had pulmonary TB and two others with extrapulmonary TB (pericarditis and brain abscess each). All four patients with active TB were treated with standard anti TB medications. Three had complete resolution of their TB and one died.

Conclusion: Baseline screening has been effectively carried out in our cohort as per recommendations. Physician should be vigilant not only for reactivation of latent TB but occurrence of de novo TB in patients on biological therapy.

\section{REFERENCES:}

[1] Gardam, M. A. et al. Anti-tumour necrosis factor agents and tuberculosis risk: mechanisms of action and clinical management. Lancet. Infect. Dis. 3, 148155, doi:10.1016/s1473-3099(03)00545-0 (2003).

[2] Winthrop, K. L., Yamashita, S., Beekmann, S. E. \& Polgreen, P. M. Mycobacterial and other serious infections in patients receiving anti-tumor necrosis factor and other newly approved biologic therapies: case finding through the Emerging Infections Network. Clin. Infect. Dis. 46, 1738-1740, doi:10.1086/587989 (2008)

[3] Cantini, F., Niccoli, L. \& Goletti, D. Tuberculosis risk in patients treated with non-anti-tumor necrosis factor- $\alpha$ (TNF- $\alpha$ ) targeted biologics and recently licensed TNF- $\alpha$ inhibitors: data from clinical trials and national registries. $J$ Rheumatol. Suppl. 91, 56-64, doi:10.3899/jrheum.140103 (2014). 
Acknowledgements: We would acknowledge Dr. Noran Alhashmi, Dr. Roaa Jodah, and Dr. Lamis Ramadan for their assistance in data collection.

Disclosure of Interests: None declared

DOI: 10.1136/annrheumdis-2021-eular.1617

\section{POS1154 CHARACTERISTICS OF SURGICALLY TREATED TUBERCULOUS AND BRUCELLAR SPONDYLODISCITIS}

D. Khalifa ${ }^{1}$, N. El Fani ${ }^{2}$, R. Moncer ${ }^{2}$, E. Toulgui ${ }^{2}$, W. Ouanes ${ }^{2}$, S. Jemni3. ${ }^{1}$ Farhat hached hospital, rheumatology, sousse, Tunisia; ${ }^{2}$ sahloul hospital, physical therapy and rehabilitation, sousse, Tunisia; ${ }^{2}$ sahloul hospital, physical therapy and rehabilitation, sousse, Tunisia

Background: Although rare in most developed countries, tuberculous and brucellar spondylodiscitis are still frequent in the mediterranean countries. Management of spondylodiscitis may require a surgical intervention in cases of spinal cord or root compression, bone destructions and deformities, unsuccessfull medical treatment or large abcesses.

Objectives: To study characteristics and outcome of surgically treated tuberculous and brucellar spondylodiscitis.

Methods: Medical records of patients who were admitted into the physical therapy and rehabilitation ward immediately post surgery of the spine were retrospectively studied. The study included records from January 2010 to December 2020.

Results: Twenty patients were diagnosed with tuberculous or brucellar spondylodiscitis for which they underwent surgery. The mean age of diagnosis was $49.7 \pm 11.9$ years. The sex ratio was $1: 1$. The diagnosis was delayed in $60 \%$ of the cases due to atypical presentations. Fatigue was reported in $45 \%$ of the patients, back pain in $60 \%$ of the cases, radicular pain in $15 \%$ of the cases, fever in $20 \%$ of the patients and abnormal gait in $60 \%$ of the cases. Tuberculosis was identified in $75 \%$ of cases and brucellar infection in $25 \%$ of the cases. On biology, elevated inflammatory markers were noted in $60 \%$ of the cases. Intradermal reaction for tuberculosis was positive in 9 patients while cultures allowed the diagnosis of tuberculosis in 1 patient. Wright serology test confirmed the diagnosis of brucellar infection in all 5 cases. In the remaing ones, vertebral biopsy or intraoperative samples allowed the diagnosis of the tuberculous spondylodiscitis. Cervical spine involvement was noted in $5 \%$ of cases, lumbar spine in $25 \%$ and dorsal spine in $30 \%$ of the cases. All patients recieved antibiotics and spinal immobilization. Surgery was indicated in $78.6 \%$ of cases for spinal cord decompression and in $21.4 \%$ of the cases for voluminous abcesses. Anterior approach was used in $7.1 \%$ of the cases and posterior approach in $92.9 \%$ of cases. $23.1 \%$ of the patients benifited concomitantly with spinal fusion surgery. Post operative success was immediately confirmed by recovery of normal gait in $15.4 \%$ of the patients. Complications after surgery were reported in $35.7 \%$ of the cases. They consisted in spinal instability in $60 \%$ of the cases, abcesses in $20 \%$ of the cases and the emergence of secondary articular localizations in $20 \%$ of the cases. $28.6 \%$ of the patients underwent a second surgery mainly to stabalize the spine. Before surgery, walking was impossible for $65 \%$ of the patients and 12 months after surgery only $30 \%$ of the patients couldn't walk ( $p=0.001) .15 .8 \%$ of the patients developed later on pressure ulcers and $45 \%$ of the patients were prescribed appropriate wheelchairs.

Conclusion: Spinal surgery in tuberculous or brucellar spondylitis may be indicated if medical treatment is insufficient and especially if neurological complications occur. Surgery may be invasive with various complications but sometimes it could be the only option to treat a neurological deficit.

REFERENCES:

[1] Guerado E, Cerván AM. Surgical treatment of spondylodiscitis. An update. Int Orthop 2012; 36: 413-420.

Disclosure of Interests: None declared.

DOI: 10.1136/annrheumdis-2021-eular.1932

\section{POS1155 INFECTIOUS RISK DURING BIOLOGIC THERAPY FOR INFLAMMATORY RHEUMATIC DISEASES: DATA FROM THE TUNISIAN BINAR REGISTRY}

S. Boussaid ${ }^{1}$, R. Ben Aissa ${ }^{2}$, S. Kochbati ${ }^{2}$, M. Elleuch ${ }^{1}$, L. Abdelmoula ${ }^{3}$, A. Laatar ${ }^{4}$, W. Hamdi ${ }^{5}$, I. Gharsallah ${ }^{6}$, E. Bouagina ${ }^{7}$, S. Baklouti ${ }^{8}$, N. Bergaoui ${ }^{9}$ M. Younes ${ }^{10} .{ }^{1}$ Rabta Hospital, Department of Rheumatology, Tunis, Tunisia; ${ }^{2}$ Habib Thameur Hospital, Department of Internal Medecine, Tunis, Tunisia; ${ }^{3}$ Charles Nicolle Hospital, Department of Rheumatology, Tunis, Tunisia; ${ }^{4}$ Mongi Slim Hospital, Department of Rheumatology, Marsa, Tunisia; ${ }^{5} \mathrm{Med}$ Taieb Kassab Institute, Department of Rheumatology, Mannouba, Tunisia; ${ }^{6}$ Military Hospital, Department of Internal Medecine, Tunis, Tunisia; ${ }^{7}$ Farhat Hachad Hospital, Department of Rheumatology, Sousse, Tunisia; ${ }^{8} \mathrm{Hedi}$ Chaker Hospital, Department of Rheumatology, Sfax, Tunisia; ${ }^{9}$ Fattouma Bourguiba Hospital, Department of Rheumatology, Monastir, Tunisia; ${ }^{10}$ Tahar Sfar Hospital, Department of Internal Medecine, Mahdia, Tunisia
Background: The development of biologics for the treatment of systemic rheumatic diseases increased the risk of infections. The management of this complication deserves particular attention since it remains a major cause of morbidity and mortality.

Objectives: The aim of our study was to determine infection frequency under biological treatment and consequences on the therapeutic management.

Methods: Patients included in the Biological National Registry (BINAR) from 2016 to 2020. Data related to the disease, biological agents, and infections occurring under biologic disease-modifying antirheumatic drugs (bDMARDs) were collected. Results: The study included 298 patients with a mean age of 49.2 years [18-79] 175 patients with rheumatoid arthritis and 123 with spondyloarthritis (Axial Spondyloarthritis $=48$, Enteropathic Arthritis $=41$, Psoriatic Arthritis=34). Anti Tumor necrosis factor-alpha (Anti-TNF) agents were the most prescribed bDMARDs in $87.9 \%(n=263)$ of patients: Infliximab $20.4 \%(n=61)$,Etanercept $23.1 \%(n=69)$ Adalimumab $24.6 \%(n=74)$ and Certolizumab $(n=79)$. No patients were treated with Golimumab. Tocilizumab and Rituximab were prescribed respectively in $10.4 \%(n=31)$ and $5 \%(n=15)$ of patients. Infections occured in 9 patients $(3.1 \%)$ with a total of 13 infectious episodes 12 bacterial and a viral one. The site of infections was: respiratory $(38 \%)$, urinary $(15 \%)$, cutaneous $(23 \%)$, ORL $(8 \%)$, infective endocarditis (8\%), and other ( $8 \%$ ). The infectious agent was identified in only 3 patients. The outcomes were favorable in most cases except in one patient where there was a definitive interruption of bDMARDs. The patient was hospitalized for sepsis complicating a cutaneous infection with favorable outcomes under antibiotics within a week. The biological agent with higher risk of infections was Tocilizumab $(p=0.056)$, unlike Rituximab $(p=0.483)$ and Anti-TNF $(p=0.082)$. All patients who had an infectious episode were under corticosteroids.

Conclusion: Our results confirm that bDMARDs are predisposing to infections, but data from BINAR showed that most infections were trivial with no serious outcomes. Therefore, infections should be assessed in patients under bDMARDs for an early therapeutic intervention.

Disclosure of Interests: None declared.

DOI: 10.1136/annrheumdis-2021-eular.3000

\section{POS1156 INFECTIONS AND OTHER ADVERSE EFFECTS WITH BIOLOGICAL THERAPIES OVER THE PAST 10 YEARS}

G. Alvarez ${ }^{1}$, A. Perez Jaen ${ }^{1}$, A. Uribe Luna ${ }^{1}$, M. Rexach Fumaña ${ }^{1}$, L. Peries Reverter $^{2}$, C. Diez Vallejo ${ }^{3}$, L. Viñas Sague ${ }^{3}$, A. Castro Guardiola ${ }^{1} .{ }^{1}$ Hospital Universitario de Girona Doctor Josep Trueta, Internal Medicine, Girona, Spain; ${ }^{2}$ Hospital Universitario de Girona Doctor Josep Trueta, Gastroenterology, Girona, Spain; ${ }^{3}$ Hospital Universitario de Girona Doctor Josep Trueta, Pharmacology, Girona, Spain

Background: Biological therapies have marked a difference in the treatment of many autoimmune conditions. As all treatments, they have their side effects although little evidence of long-term effects has been reported.

Objectives: Analyze retrospectively the appearance of infections that required hospitalization and other side effects in patients treated with biological therapies over the past 10 years.

Methods: Biological drugs dispensed in our center to non-cancer patients from 2008 to 2018 were reviewed. Health records were collected from our database and all statistical analyzes were performed with the SPSS program.

Results: 24 different biological drugs applied to 34 medical conditions; 847 treatments were dispensed over a total of 555 patients. The median age was 44 years with a mean duration of 3.6 years of treatment. The most commonly used drug were adalimumab $(n=280,33 \%)$, infliximab $(n=119,12 \%)$, etanercept $(n=$ $97.13 \%)$, rituximab $(n=63,7 \%)$, vedolizumab $(n=47,5.5 \%)$ and omalizumab ( $n$ $=43.5 \%$ ). The rest of the drugs were administered $<30$ times each (ustekinumab, golimumab, certolizumab, tocilizumab, secukinumab and abatacept), representing $<4 \%$ of the total sample.

The underlying conditions included Crohn's Disease $(n=262,31 \%)$, rheumatoid arthritis $(n=133,16 \%)$, ulcerative colitis $(n=118,14 \%)$, spondyloarthropathies $(n=86,10 \%)$, psoriatic arthritis $(n=56,7 \%)$, psoriasis $(n=49,6 \%)$, asthma $(n=$ $34,4 \%)$, nephropathies $(n=19,2 \%)$ and vasculitis $(n=13,1.5 \%)$. The rest were pathologies with $<10$ cases.

During treatment, infection requiring hospitalization occurred in $10.2 \%(n=86)$ The most frequent focus of infection was respiratory $(n=23,27 \%)$, abdominal ( $n=19,22 \%)$, soft tissue-bone $(n=17$ cases, $20 \%)$, urinary $(n=7,8 \%)$ and tuberculosis $(n=4,5 \%)$. No case of hepatitis reactivation was observed. At the time of hospital admission, $46 \%$ of patients were only under biological therapy, $21 \%$ had another non-corticosteroid immunosuppressant, $17 \%$ were associated with corticosteroid, and $15 \%$ had triple therapy (corticosteroids, another immunosuppressant and the biological drug). The drugs associated with more infections were: abatacept $20 \%$, rituximab $16 \%$ and adalimumab $13 \%$.

During the study period, 14 deaths $(1.7 \%)$ were observed; being the cause cancer-related $(n=5)$, infection $(n=5)$, the disease itself for which was receiving biological treatment $(n=2)$, and endocrine metabolic causes $(n=2)$. 